\title{
The Gamma Generalized Pareto Distribution with Applications in Survival Analysis
}

\author{
Thiago A. N. de Andrade ${ }^{1}$, Luz Milena Zea Fernandez ${ }^{2}$, Frank Gomes-Silva ${ }^{3}$ \& Gauss M. Cordeiro ${ }^{1}$ \\ ${ }^{1}$ Department of Statistics, Federal University of Pernambuco, Recife, Brazil \\ 2 Department of Statistics, Federal University of Rio Grande do Norte, Natal, Brazil \\ ${ }^{3}$ Department of Statistics and Informatics, Rural Federal University of Pernambuco, Recife, Brazil \\ Correspondence: Thiago A. N. de Andrade, Department of Statistics, Federal University of Pernambuco, Recife, Brazil. \\ Tel: 55(81)-997-793-141. E-mail: thiagoan.andrade@gmail.com
}

Received: March 15, 2017 Accepted: April 14, 2017 Online Published: April 19, 2017

doi:10.5539/ijsp.v6n3p141 URL: https://doi.org/10.5539/ijsp.v6n3p141

\begin{abstract}
We study a three-parameter model named the gamma generalized Pareto distribution. This distribution extends the generalized Pareto model, which has many applications in areas such as insurance, reliability, finance and many others. We derive some of its characterizations and mathematical properties including explicit expressions for the density and quantile functions, ordinary and incomplete moments, mean deviations, Bonferroni and Lorenz curves, generating function, Rényi entropy and order statistics. We discuss the estimation of the model parameters by maximum likelihood. A small Monte Carlo simulation study and two applications to real data are presented. We hope that this distribution may be useful for modeling survival and reliability data.
\end{abstract}

Keywords: generalized pareto distribution, hazard rate function, survival function

\section{Introduction}

The Pareto distribution is a very known statistical model, widely used for accommodate heavy-tailed distributions and many of its applications in areas such as economics, biology and physics can be found in the literature (Alzaatreh, A., et al., 2012). An important extension of the Pareto model is the generalized Pareto ( $\mathcal{P P}$ for short) distribution. It is believed that this distribution was pioneered by Pickands, J.(1975) in the extreme values context as the distribution of the sample of surpluses above a certain high level. Some of its applications are addressed to areas such as insurance, reliability, finance, meteorology and environment, among others (De Zea Bermudez, P. \& Kotz, S., 2010). In a recent paper, (Nadarajah, S. \& Gupta, A. K., 2007) list many practical situations in which the $\mathcal{G P}$ distribution has been applied. Here, we mention some of these: lifetime data analysis, coupon collector's problem, analysis of radio audience data, analysis of rainfall time series, regional flood frequency analysis, drought modeling, value at risk and measuring liquidity risk of open-end funds, among others.

The cumulative distribution function (cdf) of the $\mathcal{G P}$ distribution is given by

$$
G(x ; \xi, \sigma)= \begin{cases}1-(1+\xi x / \sigma)^{-1 / \xi}, & \text { if } \xi \neq 0 \\ 1-\exp (-x / \sigma), & \text { if } \xi=0 .\end{cases}
$$

The corresponding probability density function (pdf) is given by

$$
g(x ; \xi, \sigma)= \begin{cases}1 / \sigma(1+\xi x / \sigma)^{-1 / \xi-1}, & \text { if } \xi \neq 0 \\ 1 / \sigma \exp (-x / \sigma), & \text { if } \xi=0,\end{cases}
$$

where $\xi \in \mathbb{R}$ and $\sigma>0$ are the shape and scale parameters, respectively. The support of $g(x ; \xi, \sigma)$ is $x>0$ for $\xi \geq 0$ and $0<x<-\sigma / \xi$ for $\xi<0$.

Here, we adopt the same parametrization considered by (Song, J. \& Song, S., 2012) for the GP distribution. The case $\xi=0$ reduces to the exponential distribution. In general, it is considered that the scale parameter has a direct effect on the tails of this distribution, so that it has heavy-tailed when $\xi>0$, medium-tailed when $\xi=0$, and short-tailed when $\xi<0$ (Song, J. \& Song, S., 2012).

In recent years, several studies on generalizations of continuous distributions by introducing new parameters have been considered. Particularly, great attention has been given to generalizations by using special generators (Tahir, M. \& Nadarajah, S., 2013). In this context, some classes well-established in the literature are: the Marshall-Olkin class proposed by 
(Marshall, A. W. \& Olkin, I., 1997), the beta class pioneered by (Eugene, N., et al., 2002), the gamma class proposed by (Zografos, K. \& Balakrishnan, N., 2009) and (Ristić, M. M. \& Balakrishnan, N., 2012) and the Kumaraswamy class defined by (Cordeiro, G. M. \& De Castro, M., 2011).

For a given parent distribution with cdf $G(x)$ and pdf $g(x), x \in \mathbb{R}$, (Zografos, K. \& Balakrishnan, N., 2009) proposed the gamma- $G$ family with an extra shape parameter $a>0$ and cdf $F(x)$ given by

$$
F(x ; a)=\frac{\gamma\{a,-\log [1-G(x)]\}}{\Gamma(a)},
$$

where

$$
\gamma(a, z)=\int_{0}^{z} t^{a-1} \mathrm{e}^{-t} \mathrm{~d} t
$$

denotes the lower incomplete gamma function and $\Gamma(\cdot)$ is the gamma function. The pdf of this family is given by

$$
f(x ; a)=\frac{1}{\Gamma(a)}\{-\log [1-G(x)]\}^{a-1} g(x) .
$$

Its hazard rate function (hrf) can be expressed as

$$
\tau(x ; a)=\frac{\{-\log [1-G(x)]\}^{a-1} g(x)}{\Gamma\{a,-\log [1-G(x)]\}},
$$

where

$$
\Gamma(a, z)=\int_{z}^{\infty} t^{a-1} \mathrm{e}^{-t} \mathrm{~d} t
$$

denotes the upper incomplete gamma function. Note that $\Gamma(a)=\gamma(a, z)+\Gamma(a, z)$. Several mathematical properties about this class were studied by Nadarajah, S. (2013).

Due to its wide applicability, some recent works extended the $\mathcal{G P}$ distribution using special generators to obtain more flexibility. Two important extensions are the beta $\mathcal{G P}$ and Kumaraswamy $\mathcal{G P}$ distributions investigated by ( Mahmoudi, E., 2011) and (Nadarajah, S. \& Eljabri, S., 2013), respectively. Based on a similar motivation, we study here another promising extension of the $G \mathcal{P}$ distribution using the gamma generator called the gamma generalized Pareto (GGP for short) distribution. We present some of its mathematical properties and emphasize its application in the context of survival analysis.

The paper is organized as follows. In Section, we provide the cdf, pdf and hrf of the GGP distribution. Some important results about transformations involving this distribution are addressed in Section. Some of its mathematical properties are derived in Section including a mixture representation for its pdf and explicit expressions for the quantile function (qf), ordinary and incomplete moments, mean deviations, Bonferroni and Lorenz curves, generating function, Rényi entropy and order statistics. In Section, we use the maximum likelihood method to estimate the parameters of the $G \mathcal{G P}$ distribution. A simulation study and two applications are addressed in Section. Section provides some concluding remarks.

\subsection{The GGP Distribution}

Inserting (1) in equation (3), it gives the cdf of the $G \mathcal{G P}$ distribution

$$
F(x ; a, \xi, \sigma)= \begin{cases}\gamma[a, 1 / \xi \log (1+\xi x / \sigma)] / \Gamma(a), & \text { if } \xi \neq 0 \\ \gamma(a, x / \sigma) / \Gamma(a), & \text { if } \xi=0\end{cases}
$$

where $\xi \in \mathbb{R}, \sigma>0$ and $a>0$. The range of $x$ is $x>0$ for $\xi \geq 0$ and $0<x<-\sigma / \xi$ for $\xi<0$.

The corresponding pdf has the form

$$
f(x ; a, \xi, \sigma)= \begin{cases}\frac{1}{\sigma \Gamma(a) \xi^{a-1}}[\log (1+\xi x / \sigma)]^{a-1}(1+\xi x / \sigma)^{-1 / \xi-1}, & \text { if } \xi \neq 0 \\ \frac{1}{\sigma^{a} \Gamma(a)} x^{a-1} \exp (-x / \sigma), & \text { if } \xi=0,\end{cases}
$$


and its hrf is given by

$$
\tau(x ; a, \xi, \sigma)= \begin{cases}\frac{[\log (1+\xi x / \sigma)]^{a-1}(1+\xi x / \sigma)^{-1 / \xi-1}}{\sigma \xi^{a-1} \Gamma[a, 1 / \xi \log (1+\xi x / \sigma)]}, & \text { if } \xi \neq 0 \\ \frac{x^{a-1} \exp (-x / \sigma)}{\sigma^{a} \Gamma(a, x / \sigma)}, & \text { if } \xi=0 .\end{cases}
$$

Setting $\xi=0$ in equation (6), we have the gamma exponential distribution, which was studied by (Risti'c, M. M. \& Balakrishnan, N., 2012) and (Nascimento, D. C., 2014), whereas setting $\xi<0$ leads to a support for $X$ that depends on unknown parameters. For this reason, we consider only the $G \mathcal{G P}$ model with $\xi>0$ and positive support. We emphasize that (Nadarajah, S.\& Eljabri, S., 2013) gave the cdf (Dahiya, R. C., and Gurland, J., 1972) outside the context of the gamma generator but they did not study any of its properties.

Henceforth, a random variable $X$ having cdf (6) is denoted by $X \sim \mathcal{G} \mathcal{G P}(a, \xi, \sigma)$ and we write $F(x)=F(x ; a, \xi, \sigma)$ in order to simplify the notation. Plots of the pdf and hrf of $X$ for selected parameter values are displayed in Figures and, respectively.

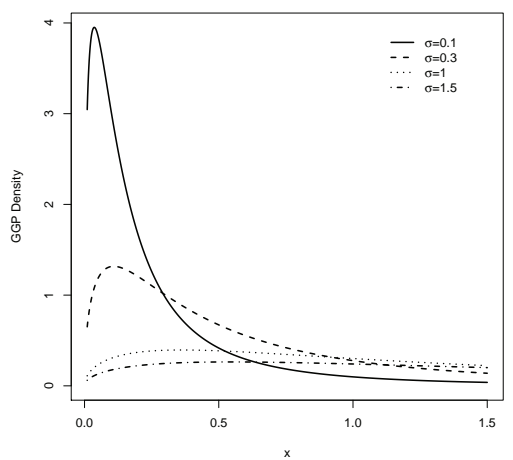

(a) $\xi=0.5$ and $a=1.5$

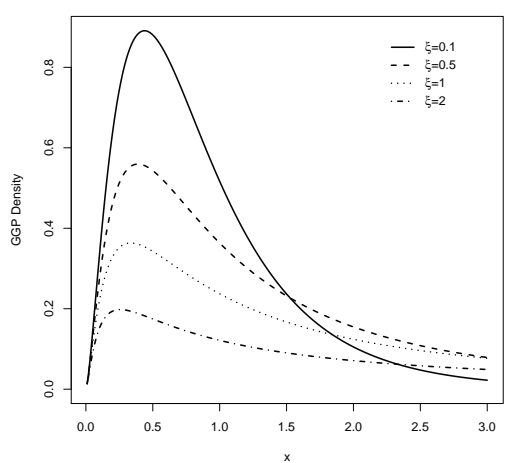

(c) $a=2.5$ and $\sigma=0.3$

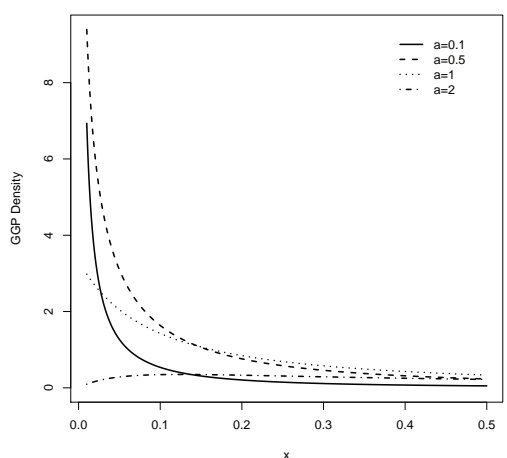

(b) $\xi=2.5$ and $\sigma=0.3$

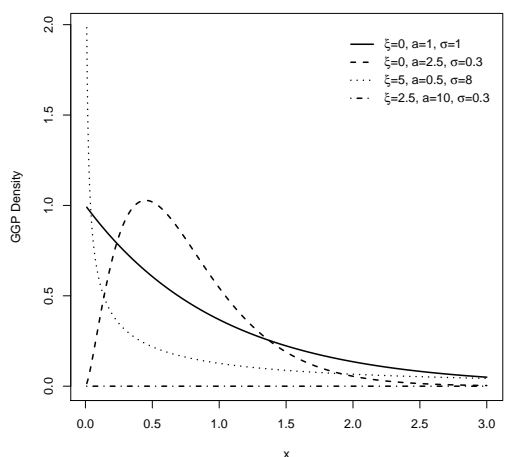

(d)

Figure 1. Plots of the $\mathcal{G} \mathcal{G P}$ density function for some parameter values.

\section{Some Important Results}

Theorem 2.1 Let $X \sim \mathcal{G G P}(a, \xi, \sigma)$, where $a, \xi$ and $\sigma$ are positive real numbers. Then, the random variable $Y=$ $\log [\log (1+\xi X / \sigma)]$ has the $\log$-gamma distribution with parameters $a$ and $1 / \xi$, namely $Y \sim \log$-gamma $(a, 1 / \xi)$.

Proof. Consider the function $h(x)=\log [\log (1+\xi x / \sigma)]$ and its inverse function $h^{-1}(x) \quad=$ $\sigma\left[\exp \left(\mathrm{e}^{x}\right)-1\right] / \xi$. The density function of $Y$, say $f_{Y}(y)$, is obtained from the transformation method and equation (7) gives

$$
f_{Y}(y)=\frac{1}{\Gamma(a) \xi^{a}}\left(\mathrm{e}^{y}\right)^{a-1} \exp \left(\mathrm{e}^{y}+y\right)\left[\exp \left(\mathrm{e}^{y}\right)\right]^{-1 / \xi-1}
$$




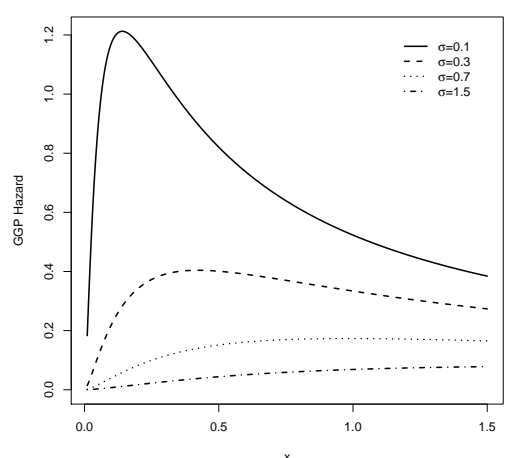

(e) $\xi=1$ and $a=2.5$

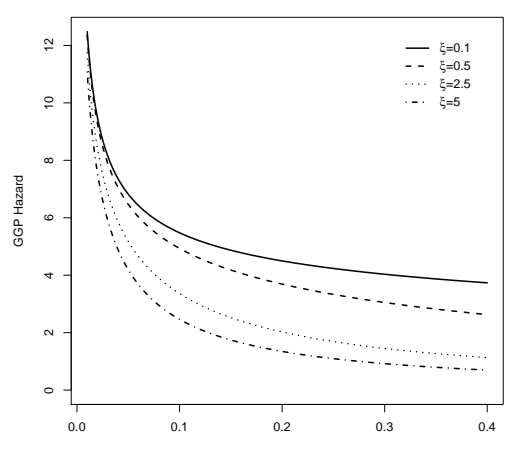

(g) $a=0.5$ and $\sigma=0.3$

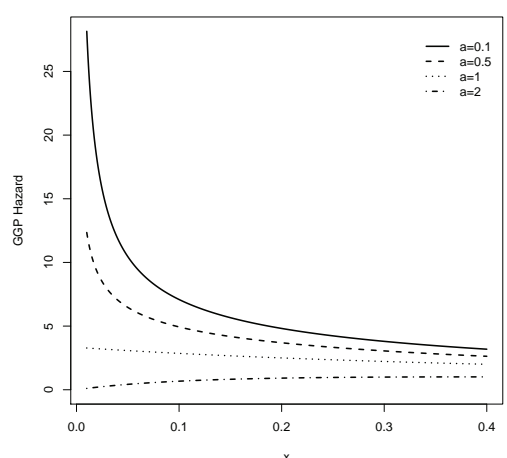

(f) $\xi=0.5$ and $\sigma=0.3$

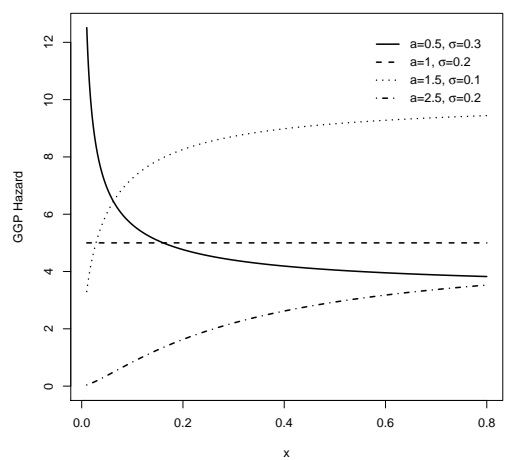

(h) $\xi=0$

Figure 2. Plots of the $\mathcal{G} \mathcal{G P}$ hrf for some parameter values.

$$
=\frac{1}{\Gamma(a) \xi^{a}} \mathrm{e}^{y a} \exp \left(-\mathrm{e}^{y} / \xi\right) .
$$

Further, if $W \sim \operatorname{gamma}(a, 1 / \xi)$, then $\log (W) \sim \log$-gamma $(a, 1 / \xi)$ and its density function agrees with the last equation.

In the context of survival analysis, the Weibull distribution is commonly used to model data with monotone hrf. An important generalization of the Weibull model is the gamma-exponentiated Weibull (GEW) distribution proposed by (Pinho, L. G., et al., 2012). The GEW model can be characterized as follows.

Theorem 2.2 Let $X$ be a random variable such that $X \sim \mathcal{G G P}(a, \xi, \sigma)$, where $a, \xi$ and $\sigma$ are positive real numbers. Then, the random variable

$$
Y=-\xi \log \left(\frac{\xi X / \sigma}{1+\xi X / \sigma}\right)
$$

has the GEW distribution proposed by (Pinho, L. G., et al., 2012) with parameters $\delta=a, \alpha=1 / \xi, k=1$ and $\lambda=\xi$. Using their notation, we write $Y \sim \operatorname{GEW}(a, 1 / \xi, 1, \xi)$.

Proof. Consider the function $h(x)=-\xi \log \left(\frac{\xi x / \sigma}{1+\xi x / \sigma}\right)$. It is easy to obtain

$$
h^{-1}(x)=\frac{\sigma \mathrm{e}^{-x / \xi}}{\xi\left(1-\mathrm{e}^{-x / \xi}\right)} \quad \text { and } \quad \frac{\mathrm{d} h^{-1}(x)}{\mathrm{d} x}=-\frac{\sigma \mathrm{e}^{-x / \xi}}{\xi^{2}\left(1-\mathrm{e}^{-x / \xi}\right)^{2}} .
$$

From equation (7) and using the transformation method, the density function of $Y, f_{Y}(y)$, can be expressed as

$$
f_{Y}(y)=\frac{1}{\Gamma(a) \xi^{a+1}} \mathrm{e}^{-y / \xi}\left(1-\mathrm{e}^{-y / \xi}\right)^{1 / \xi-1}\left[-\log \left(1-\mathrm{e}^{-y / \xi}\right)\right]^{a-1} .
$$

The last equation agrees with equation (5) of (Pinho, L. G., et al., 2012) by taking $\delta=a, \alpha=1 / \xi, k=1$ and $\lambda=\xi$. 
Theorem 2.3 [Transformation] Let $Y$ be a gamma distributed random variable with parameters $a$ and $1 / \xi$. Then, the random variable

$$
X=\sigma\left(\mathrm{e}^{Y}-1\right) / \xi
$$

has the $\mathcal{G} \mathcal{P P}$ distribution with parameters $a, \xi$ and $\sigma$.

\section{Mathematical Properties}

Many structural properties of the $\mathcal{G G P}$ distribution can be derived using the concept of exponentiated distributions. The class of exponentiated distributions was initially studied by (Lehmann, E. L., 1953), who defined the so-called Lehmann alternatives. However, this class has received special attention in the literature over the last twenty years and several papers have been published. An excellent review of these publications is provided by (Tahir, M. \& Nadarajah, S., 2013). Here, we refer to the papers: (Mudholkar, G. S. \& Srivastava, D. K., 1993) for exponentiated Weibull, (Gupta, R., et al., 1998) for exponentiated Pareto, (Gupta, R. D. \& Kundu, D., 2001) for exponentiated exponential, (Nadarajah, S. \& Kotz, S., 2003) for exponentiated Fréchet, (Nadarajah, S. \& Gupta, A. K., 2007) for exponentiated gamma, (Carrasco, J. M. F., 2008) for exponentiated modified Weibull and (Lemonte, A. J. \& Cordeiro, G. M., 2011) for exponentiated generalized inverse Gaussian distribution.

\subsection{A Useful Representation}

For any baseline cdf $G(x)$, a random variable $Y$ is said to have the exponentiated- $G$ ("exp- $G$ " for short) distribution with power parameter $\alpha>0$, say $Y \sim G^{\alpha}$, if its cdf and pdf are given by $H_{\alpha}(x)=G(x)^{\alpha}$ and $h_{\alpha}(x)=\alpha g(x) G(x)^{\alpha-1}$, respectively. Using a result pioneered by (Nadarajah, S., et al., 2013), we can express the density of the $G \mathcal{G P}$ distribution in terms of $\exp -\mathcal{G P}$ densities. Thus, for a real non-integer $a>0$, equation (7) can be expressed as

$$
f(x)=\sum_{k=0}^{\infty} b_{k} h_{a+k}(x)
$$

where, the coefficients $b_{k}$ are given by

$$
b_{k}=\frac{1}{(a+k) \Gamma(a-1)}\left(\begin{array}{c}
k+1-a \\
k
\end{array}\right) \sum_{j=0}^{k} \frac{(-1)^{j+k} p_{j, k}}{(a-1-j)}\left(\begin{array}{c}
k \\
j
\end{array}\right) \text {, }
$$

and the constants $p_{j, k}$ can be determined recursively by

$$
p_{j, k}=k^{-1} \sum_{m=1}^{k} \frac{(-1)^{m}[m(j+1)-k]}{(m+1)} p_{j, k-m},
$$

for $k=1,2, \ldots$ and $p_{j, 0}=1$. Here, $h_{a+k}(x)$ denotes the exp-GP density with power parameter $a+k$ given by

$$
h_{a+k}(x)=\frac{(a+k)}{\sigma}(1+\xi x / \sigma)^{-1 / \xi-1}\left[1-(1+\xi x / \sigma)^{-1 / \xi}\right]^{a+k-1} .
$$

Equation (9) reveals that the $\mathcal{G} \mathcal{G P}$ density function is a linear combination of exp- $\mathcal{G P}$ densities. This result is important to derive some mathematical properties of $X$ such as the ordinary and incomplete moments, generating function and mean deviations from those of the exp-GP distribution.

\subsection{Quantile Function}

The qf is obtained as the inverse of the cumulative function. It follows from equation (6) that the qf of the $\mathcal{G} \mathcal{G P}$ distribution can be expressed as

$$
x_{u}=Q(u)=\frac{\sigma}{\xi}\left\{\exp \left[\xi W^{-1}(a, u)\right]-1\right\},
$$

for $0<u<1$, where $W^{-1}(a, u)$ is the qf of the gamma distribution with shape parameter $a$ and scale parameter one evaluated at $u$. 
The function $W^{-1}(a, u)$ can be expressed as a power series expansion (Cordeiro, G. M., 2013)

$$
W^{-1}(a, u)=\sum_{i=0}^{\infty} t_{i}[\Gamma(a+1) u]^{i / a},
$$

where $t_{0}=0, t_{1}=1$ and any coefficient $t_{i+1}$ for $i \geq 1$ is determined by the cubic recurrence equation

$$
\begin{aligned}
t_{i+1}= & \frac{1}{i(a+i)}\left\{\sum_{r=1}^{i} \sum_{s=1}^{i-s+1} s(i-r-s+2) t_{r} t_{s} t_{i-r-s+2}\right. \\
& \left.-\sum_{r=2}^{i} r[r-a+(a-1)(i+2-r)] t_{r} t_{i-r+2}\right\}
\end{aligned}
$$

where $\Delta(i)=0$ if $i<2$ and $\Delta(i)=1$ if $i \geq 2$. The first few coefficients are $t_{2}=1 /(a+1), t_{3}=(3 a+5) /\left[2(a+1)^{2}(a+2)\right], \ldots$ It follows directly from (12) that the median of $X$ is simply $x_{1 / 2}=Q(1 / 2)$. Further, it is possible to generate $\mathcal{G G P}$ variates by $X=Q(U)$, where $U$ is a uniform variate on the unit interval $(0,1)$.

Another important application of the $\mathrm{qf}$ is to obtain the measures of skewness and kurtosis. In this sense, two important measures are the Bowley skewness (B) and the Moors kurtosis (M). The Bowley skewness (Kenney, J. F. \& Keeping, E. S., 1962), based on quartiles, and the Moors kurtosis (Moors, J. J., 1988), based on octiles, are given by

$$
B=\frac{Q(3 / 4)+Q(1 / 4)-2 Q(1 / 2)}{Q(3 / 4)-Q(1 / 4)} \quad \text { and } \quad M=\frac{Q(7 / 8)-Q(5 / 8)+Q(3 / 8)-Q(1 / 8)}{Q(6 / 8)-Q(2 / 8)},
$$

respectively.

\subsection{Moments}

The $n$th moment of $X$ can be determined based on (9) as

$$
E\left(X^{n}\right)=\frac{1}{\sigma} \sum_{k=0}^{\infty} b_{k}(a+k) \int_{0}^{\infty} x^{n}(1+\xi x / \sigma)^{-1 / \xi-1}\left[1-(1+\xi x / \sigma)^{-1 / \xi}\right]^{a+k-1} d x
$$

Setting $u=(1+\xi x / \sigma)^{-1 / \xi}, E\left(X^{n}\right)$ can be expressed as

$$
E\left(X^{n}\right)=\left(\frac{\sigma}{\xi}\right)^{n} \sum_{k=0}^{\infty} b_{k}(a+k) \int_{0}^{1}\left(u^{-\xi}-1\right)^{n}(1-u)^{a+k-1} d u
$$

Using the binomial expansion for $\left(u^{-\xi}-1\right)^{n}$ and the generalized binomial expansion for $(1-u)^{a+k-1}$, the $n$th moment of $X$ reduces to

$$
E\left(X^{n}\right)=\left(\frac{\sigma}{\xi}\right)^{n} \sum_{k, \ell=0}^{\infty} \sum_{i=0}^{n} \frac{(-1)^{\ell+n-i}(a+k) b_{k}}{(\ell-i \xi+1)}\left(\begin{array}{l}
n \\
i
\end{array}\right)\left(\begin{array}{c}
a+k-1 \\
\ell
\end{array}\right)
$$

\subsection{Generating Function}

The moment generating function (mgf) of $X$ can be obtained using the fact that the $\mathcal{G G P}$ density function is a linear combination of exp-GP densities. Thus,

$$
M(t)=\frac{1}{\sigma} \sum_{k=0}^{\infty} b_{k}(a+k) \int_{0}^{\infty} \mathrm{e}^{t x}(1+\xi x / \sigma)^{-1 / \xi-1}\left[1-(1+\xi x / \sigma)^{-1 / \xi}\right]^{a+k-1} d x
$$


Setting $u=(1+\xi x / \sigma)^{-1 / \xi}, M(t)$ can be expressed as

$$
M(t)=\mathrm{e}^{-\frac{t \sigma}{\xi}} \sum_{k=0}^{\infty} b_{k}(a+k) \int_{0}^{1} \exp \left(t \sigma u^{-\xi} / \xi\right)(1-u)^{a+k-1} d u .
$$

Using the generalized binomial expansion for $(1-u)^{a+k-1}, M(t)$ reduces to

$$
M(t)=\mathrm{e}^{-\frac{t \sigma}{\xi}} \sum_{k, i=0}^{\infty}(-1)^{i}(a+k) b_{k}\left(\begin{array}{c}
a+k-1 \\
i
\end{array}\right) \int_{0}^{1} \exp \left(t \sigma u^{-\xi} / \xi\right) u^{i} d u
$$

Thus, it follows that (for $t<0$ )

$$
M(t)=\frac{\mathrm{e}^{-\frac{t \sigma}{\xi}}}{\xi} \sum_{k, i=0}^{\infty}(-1)^{i}(a+k) b_{k}\left(\begin{array}{c}
a+k-1 \\
i
\end{array}\right)\left(-\frac{\xi}{t \sigma}\right)^{-(\ell+1) / \sigma} \Gamma\left[-\frac{(1+i)}{\xi},-\frac{t \sigma}{\xi}\right] .
$$

\subsection{Incomplete Moments}

Frequently, in applied works it is of interest to know the $n$th incomplete moment of a random variable. The $n$th incomplete moment of $X$ is given by $T_{n}(z)=\int_{-\infty}^{z} x^{n} f(x) d x$, and using equation (9), we can write

$$
T_{n}(z)=\frac{1}{\sigma} \sum_{k=0}^{\infty} b_{k}(a+k) \int_{0}^{z} x^{n}(1+\xi x / \sigma)^{-1 / \xi-1}\left[1-(1+\xi x / \sigma)^{-1 / \xi}\right]^{a+k-1} d x
$$

Setting $u=(1+\xi x / \sigma)^{-1 / \xi}, T_{n}(z)$ reduces to

$$
T_{n}(z)=\left(\frac{\sigma}{\xi}\right)^{n} \sum_{k=0}^{\infty} b_{k}(a+k) \int_{t}^{1}\left(u^{-\xi}-1\right)^{n}(1-u)^{a+k-1} d u,
$$

where $t=(1+\xi z / \sigma)^{-1 / \xi}$.

Using the binomial expansion for $\left(u^{-\xi}-1\right)^{n}$ and the generalized binomial expansion for $(1-u)^{a+k-1}, T_{n}(z)$ reduces to

$$
T_{n}(z)=\left(\frac{\sigma}{\xi}\right)^{n} \sum_{k, \ell=0}^{\infty} \sum_{i=0}^{n}(-1)^{\ell+n-i}(a+k) b_{k}\left(\frac{1-t^{\ell-i \xi+1}}{\ell-i \xi+1}\right)\left(\begin{array}{c}
n \\
i
\end{array}\right)\left(\begin{array}{c}
a+k-1 \\
\ell
\end{array}\right)
$$

A particularly important case is the first incomplete moment. From equation (13), we have

$$
T_{1}(z)=\frac{\sigma}{\xi} \sum_{k, \ell=0}^{\infty}(-1)^{\ell}(a+k) b_{k}\left(\frac{1-t^{\ell-\xi+1}}{\ell-\xi+1}-\frac{1-t^{\ell+1}}{\ell+1}\right)\left(\begin{array}{c}
a+k-1 \\
\ell
\end{array}\right)
$$

An important application of equation (14) refers to the mean deviations of $X$ about the mean and the median. These quantities are defined by

$$
\delta_{1}(X)=2 \mu_{1}^{\prime} F\left(\mu_{1}^{\prime}\right)-2 T_{1}\left(\mu_{1}^{\prime}\right) \quad \text { and } \quad \delta_{2}(X)=\mu_{1}^{\prime}-2 T_{1}\left(x_{1 / 2}\right) \text {, }
$$


respectively, where $\mu_{1}^{\prime}=E(X), x_{1 / 2}$ is the median of $X$ that follows from (12) as $x_{1 / 2}=Q(1 / 2), F\left(\mu_{1}^{\prime}\right)$ is easily obtained from (6) and the quantities $T_{1}\left(\mu_{1}^{\prime}\right)$ and $T_{1}\left(x_{1 / 2}\right)$ are given by (14).

Equation (14) can also be used to determine the Bonferroni and Lorenz curves, which have several applications in economics, reliability, demography and medicine. These curves are defined by $B(\pi)=T_{1}(q) /\left(\pi \mu_{1}^{\prime}\right)$ and $L(\pi)=T_{1}(q) / \mu_{1}^{\prime}$, where $q=Q(\pi)$ is calculated by (12) for a given probability $\pi$.

\subsection{Rényi Entropy}

The Rényi entropy of $X$ is given by

$$
I_{R}(\gamma)=\log \left(\int_{0}^{\infty} f^{\gamma}(x) \mathrm{d} x\right)
$$

where $\gamma>0, \gamma \neq 1$ and $f(x)$ is the pdf (7).

For a general cdf $G(x)$ satisfying equation (3), (Nadarajah, S., et al., 2013) proved that

$$
\int_{0}^{\infty} f^{\gamma}(x) \mathrm{d} x=\frac{\gamma(a-1)}{\Gamma^{\gamma}(a)} \sum_{k=0}^{\infty}\left(\begin{array}{c}
k-\gamma(a-1) \\
k
\end{array}\right) \sum_{j=0}^{k} \frac{(-1)^{j+k} p_{j, k}}{[\gamma(a-1)-j]}\left(\begin{array}{l}
k \\
j
\end{array}\right) \mathcal{I}_{k},
$$

where

$$
\mathcal{I}_{k}=\int_{0}^{\infty} G(x)^{\gamma(a-1)+k} g^{\gamma}(x) \mathrm{d} x
$$

and $p_{j, k}$ is defined by (10).

For the $\mathcal{G P}$ cdf, the integral given in equation (17) becomes

$$
\begin{aligned}
\mathcal{I}_{k} & =\int_{0}^{\infty}\left[1-(1+\xi x / \sigma)^{-1 / \xi}\right]^{\gamma(a-1)+k}\left[1 / \sigma(1+\xi x / \sigma)^{-1 / \xi-1}\right]^{\gamma} \mathrm{d} x \\
& =\frac{1}{\sigma^{\gamma}} \int_{0}^{\infty}(1+\xi x / \sigma)^{-\gamma(1 / \xi+1)}\left[1-(1+\xi x / \sigma)^{-1 / \xi}\right]^{\gamma(a-1)+k} \mathrm{~d} x .
\end{aligned}
$$

Setting $u=(1+\xi x / \sigma)^{-1 / \xi}$, the integral $\mathcal{I}_{k}$ can be expressed as

$$
\begin{aligned}
\mathcal{I}_{k} & =\frac{1}{\sigma^{\gamma-1}} \int_{0}^{1}(1-u)^{\gamma(a-1)+k} u^{(\xi+1)(\gamma-1)} \mathrm{d} u \\
& =\frac{1}{\sigma^{\gamma-1}} \sum_{\ell=0}^{\infty} \frac{(-1)^{\ell}}{\ell+\xi(\gamma-1)+\gamma}\left(\begin{array}{c}
\gamma(a-1)+k \\
\ell
\end{array}\right),
\end{aligned}
$$

whenever $\ell+\xi(\gamma-1)+\gamma \neq-1$.

Then, equation (16) can be reduced to

$$
\begin{aligned}
\int_{0}^{\infty} f^{\gamma}(x) \mathrm{d} x= & \frac{\gamma(a-1)}{\Gamma \gamma(a) \sigma^{\gamma-1}} \sum_{k=0}^{\infty}\left(\begin{array}{c}
k-\gamma(a-1) \\
k
\end{array}\right) \sum_{j=0}^{k} \frac{(-1)^{j+k} p_{j, k}}{[\gamma(a-1)-j]}\left(\begin{array}{l}
k \\
j
\end{array}\right) \\
& \times \sum_{\ell=0}^{\infty} \frac{(-1)^{\ell}}{[\ell+\xi(\gamma-1)+\gamma]}\left(\begin{array}{c}
\gamma(a-1)+k \\
\ell
\end{array}\right) \\
= & \frac{\gamma(a-1)}{\Gamma \gamma(a) \sigma^{\gamma-1}} \sum_{k, \ell=0}^{\infty} \sum_{j=0}^{k} \frac{(-1)^{j+k+\ell} p_{k, j}}{[\gamma(a-1)-j][\ell+\xi(\gamma-1)+\gamma]}\left(\begin{array}{c}
k \\
j
\end{array}\right)\left(\begin{array}{c}
\gamma(a-1)+k \\
\ell
\end{array}\right) .
\end{aligned}
$$

Then, from equations (15) and (18), the entropy of $X$ can be given as

$$
I_{R}(\gamma)=\frac{1}{1-\gamma} \log \left[\frac{\gamma(a-1)}{\Gamma^{\gamma}(a) \sigma^{\gamma-1}} \sum_{k, \ell=0}^{\infty} \sum_{j=0}^{k} \frac{(-1)^{j+k+\ell} p_{k, j}}{[\gamma(a-1)-j][\ell+\xi(\gamma-1)+\gamma]}\right.
$$




$$
\begin{gathered}
\left.\times\left(\begin{array}{l}
k \\
j
\end{array}\right)\left(\begin{array}{c}
\gamma(a-1)+k \\
\ell
\end{array}\right)\right] \\
=\frac{\log (\gamma)+\log (a-1)-\gamma \log [\Gamma(a)]-(\gamma-1) \log (\sigma)}{1-\gamma} \\
\quad+\log \left\{\sum_{k, \ell=0}^{\infty} \sum_{j=0}^{k} \frac{(-1)^{j+k+\ell} p_{k, j}}{[\gamma(a-1)-j][\ell+\xi(\gamma-1)+\gamma]}\left(\begin{array}{c}
k \\
j
\end{array}\right)\left(\begin{array}{c}
\gamma(a-1)+k \\
\ell
\end{array}\right)\right\},
\end{gathered}
$$

whenever $\ell+\xi(\gamma-1)+\gamma \neq-1$.

\subsection{Order Statistics}

Here, we derive an explicit expression for the density of the $i$ th order statistic $X_{i: n}$, say $f_{i: n}(x)$, in a random sample of size $n$ from the $\mathcal{G} \mathcal{G P}$ distribution. Using results by (Nadarajah, S., et al., 2013), we can write

$$
f_{i: n}(x)=\sum_{j=0}^{n-i} \sum_{r, k=0}^{\infty} m_{j, r, k} h_{a(j+i)+r+k}(x),
$$

where

$$
m_{j, r, k}=\frac{(-1)^{j} n !}{(i-1) !(n-i-j) ! j !} \frac{(a+r) b_{r} f_{j+i-1, k}}{[a(j+i)+r+k]},
$$

and

$$
f_{j+i-1, k}=\left(k b_{0}\right)^{-1} \sum_{m=1}^{k}[m(j+i)-k] b_{m} f_{j+i-1, k-m} .
$$

Here, $h_{a(j+i)+r+k}(x)$ denotes the pdf of the exp-GP distribution with power parameter $a(j+i)+r+k$ and it can be easily obtained by a reparameterization of (11).

Equation (19) reveals that the density of the $i$ th order statistic of the $\mathcal{G} \mathcal{G P}$ distribution is a mixture of exp- $\mathcal{G P}$ densities. This equation can be used, for example, to obtain the moments and mgf of the $\mathcal{G G P}$ order statistics.

The sth moment of $X_{i: n}$ is given by

$$
\begin{aligned}
E\left(X_{i: n}^{s}\right) & =\int_{-\infty}^{\infty} x^{s} f_{i: n}(x) d x \\
& =\sum_{j=0}^{n-i} \sum_{r, k=0}^{\infty} m_{j, r, k} \int_{0}^{\infty} x^{s} h_{a(j+i)+r+k}(x) d x .
\end{aligned}
$$

Using similar results to those presented in Section, we have

$$
\begin{aligned}
E\left(X_{i: n}^{s}\right)=\left(\frac{\sigma}{\xi}\right)^{s} & \sum_{j=0}^{n-i} \sum_{r, k, \ell=0}^{\infty} \sum_{q=0}^{s}(-1)^{\ell+s-q}\left(\begin{array}{l}
s \\
q
\end{array}\right)\left(\begin{array}{c}
a(j+i)+r+k-1 \\
\ell
\end{array}\right) \\
& \times \frac{[a(j+i)+r+k]}{(\ell-q \xi+1)} m_{j, r, k} .
\end{aligned}
$$

The mgf of $X_{i: n}$ can be expressed as 


$$
\begin{aligned}
\varphi(t) & =\int_{-\infty}^{\infty} \mathrm{e}^{t x} f_{i: n}(x) d x \\
& =\sum_{j=0}^{n-i} \sum_{r, k=0}^{\infty} m_{j, r, k} \int_{0}^{\infty} \mathrm{e}^{t x} h_{a(j+i)+r+k}(x) d x
\end{aligned}
$$

which, using similar results to those presented in Section, reduces (for $t<0$ ) to

$$
\begin{array}{r}
\varphi(t)=\frac{\mathrm{e}^{-\frac{t \sigma}{\xi}}}{\xi} \sum_{j=0}^{n-i} \sum_{r, k, \ell=0}^{\infty}(-1)^{\ell}[a(j+i)+r+k] m_{j, r, k}\left(-\frac{\xi}{t \sigma}\right)^{-(1+\ell) / \sigma} \\
\times\left(\begin{array}{c}
a(j+i)+r+k-1 \\
\ell
\end{array}\right) \Gamma\left[-\frac{(1+i)}{\xi},-\frac{t \sigma}{\xi}\right] .
\end{array}
$$

\section{Estimation}

In this section, we emphasize how to obtain the MLEs of the parameters $a, \xi$ and $\sigma$ of the $\mathcal{G} \mathcal{G P}$ distribution. Let $x_{1}, \ldots, x_{n}$ be a sample of size $n$ from this distribution. The log-likelihood function for the vector of parameters $\boldsymbol{\theta}^{\top}=(a, \xi, \sigma)^{\top}$ can be expressed as

$$
\begin{aligned}
\ell(\boldsymbol{\theta})= & -n(a-1) \log (\xi)-n \log (\sigma)-n \log [\Gamma(a)]+(a-1) \sum_{i=1}^{n} \log \left[\log \left(z_{i}\right)\right] \\
& -\left(1+\frac{1}{\xi}\right) \sum_{i=1}^{n} \log \left(z_{i}\right),
\end{aligned}
$$

where $z_{i}=1+\xi x_{i} / \sigma$.

The elements of the score vector are given by

$$
\begin{aligned}
& \frac{\partial \ell(\boldsymbol{\theta})}{\partial \xi}=-\frac{n(a-1)}{\xi}+\sum_{i=1}^{n} \log \left(z_{i}\right)-\frac{(1+\xi)}{\xi^{2}} \sum_{i=1}^{n} \frac{z_{i}-1}{z_{i}}+\frac{(a-1)}{\xi} \sum_{i=1}^{n} \frac{z_{i}-1}{z_{i} \log \left(z_{i}\right)}, \\
& \frac{\partial \ell(\boldsymbol{\theta})}{\partial \sigma}=-\frac{n}{\sigma}+\frac{(1+\xi)}{\xi \sigma} \sum_{i=1}^{n} \frac{z_{i}-1}{z_{i}}+\frac{(a-1)}{\sigma} \sum_{i=1}^{n} \frac{z_{i}-1}{z_{i} \log \left(z_{i}\right)}, \\
& \frac{\partial \ell(\boldsymbol{\theta})}{\partial a}=-n \log (\xi)-n \psi(a)+\sum_{i=1}^{n} \log \left[\log \left(z_{i}\right)\right],
\end{aligned}
$$

where $\psi(a)=\mathrm{d} \log [\Gamma(a)] / \mathrm{d} a$ and $z_{i}=1+\xi x_{i} / \sigma$.

The MLE $\widehat{\boldsymbol{\theta}}$ of $\boldsymbol{\theta}$ is obtained by solving simultaneously the nonlinear equations $U_{\dot{\xi}}(\boldsymbol{\theta})=0, U_{\sigma}(\boldsymbol{\theta})=0$ and $U_{a}(\boldsymbol{\theta})=$ 0 . These estimators can be obtained numerically by maximizing the log-likelihood function by means of algorithms for non-linear optimization. For confidence intervals and hypothesis tests, it is important to determine the observed information matrix. Particularly, for the $\mathcal{G} \mathcal{G P}$ distribution, the $3 \times 3$ observed information matrix is $J(\theta)=\left\{-U_{r s}\right\}$, where $U_{r s}=\partial^{2} \ell(\boldsymbol{\theta}) /\left(\partial \boldsymbol{\theta}_{r} \partial \boldsymbol{\theta}_{s}\right)$ for $r, s \in\{a, \xi, \sigma\}$. The elements of $\boldsymbol{J}(\boldsymbol{\theta})$ are listed in the Appendix.

\section{Applied Results}

In this section, a simulation study and two applications to real data are considered. The parameter estimation was made using the R software (www.r-project.org) by the maxLik package with simulated annealing method, which is useful for finding a global maximum when the objective function has some local maximums. 


\subsection{Simulation Study}

Here, we present the results of a Monte Carlo simulation study, which was carried out to evaluate the performance of the MLEs for the parameters $a, \xi$ and $\sigma$ of the $G G P$ distribution in finite samples. The results are obtained from 10,000 Monte Carlo replications. We take samples of sizes $n=100,200,300,500$ and 800 . In the data generation process, we consider equation (12) and the true parameters values $a=2, \xi=0.5$ and $\sigma=1.5$.

In Table 1, we provide the means, standard error estimates, root mean square errors (RMSEs) and mean absolute errors (MAEs) of $\widehat{a}, \widehat{\xi}$ and $\widehat{\sigma}$ for the GGP model. Based on the results, we can verify that the estimators are biased even for moderate samples. Thus, the use of bias-correction techniques can improve the performance of these estimators in finite samples.

Table 1. Means, standard error estimates, Biases RMSEs and MAEs of $\widehat{a}, \widehat{\xi}$ and $\widehat{\sigma}$ for the $G \mathcal{G P}^{\text {model }}$

\begin{tabular}{|c|c|c|c|c|c|c|}
\hline$n$ & Parameter & Mean & Biases & Standard Error & RMSE & MAE \\
\hline \multirow{3}{*}{100} & $a$ & 2.4407 & -0.4407 & 1.1535 & 1.2348 & 0.7749 \\
\hline & $\xi$ & 0.4630 & 0.0370 & 0.1017 & 0.1082 & 0.0826 \\
\hline & $\sigma$ & 1.5792 & -0.0792 & 1.0401 & 1.0430 & 0.8041 \\
\hline & $\bar{a}$ & $\overline{2} . \overline{2} \overline{14} \overline{3}$ & $-\overline{-0.2 \overline{1} \overline{4}}$ & $0 . \overline{7} \overline{5} 5 \overline{4}$ & $\overline{0} \overline{\overline{7}} \overline{85} \overline{2}$ & $\overline{0} . \overline{4} \overline{73} \overline{3}$ \\
\hline \multirow[t]{3}{*}{200} & $\xi$ & 0.4819 & 0.0181 & 0.0700 & 0.0723 & 0.0563 \\
\hline & $\sigma$ & 1.5289 & -0.0289 & 0.7084 & 0.7089 & 0.5549 \\
\hline & $\bar{a}$ & $\overline{2} . \overline{128} \overline{1}$ & $-0.1 \overline{2} 81$ & $0 . \overline{5} 32 \overline{1}$ & $\overline{0} . \overline{5} \overline{4} 7 \overline{3}$ & $\overline{0} . \overline{3} \overline{47} \overline{6}$ \\
\hline \multirow[t]{3}{*}{300} & $\xi$ & 0.4895 & 0.0105 & 0.0567 & 0.0577 & 0.0455 \\
\hline & $\sigma$ & 1.5126 & -0.0126 & 0.5604 & 0.5605 & 0.4419 \\
\hline & $\bar{a}$ & $\overline{2} . \overline{0} \overline{6} 6 \overline{8}$ & $-0.0 \overline{6} \overline{68}$ & $0 . \overline{3} \overline{4} 3 \overline{2}$ & $\overline{0} . \overline{3} \overline{49} \overline{6}$ & $\overline{0} . \overline{2} \overline{4} 6 \overline{3}$ \\
\hline \multirow[t]{3}{*}{500} & $\xi$ & 0.4938 & 0.0062 & 0.0445 & 0.0449 & 0.0354 \\
\hline & $\sigma$ & 1.5088 & -0.0088 & 0.4308 & 0.4309 & 0.3415 \\
\hline & $\bar{a}$ & $\overline{2} . \overline{3} \overline{7} \overline{5}$ & $-0.0 \overline{3} 75$ & $0 . \overline{4} \overline{4} \overline{0}$ & $\overline{0} \overline{2} \overline{4} 6 \overline{9}$ & $\overline{0} . \overline{1} 85 \overline{9}$ \\
\hline \multirow[t]{2}{*}{800} & $\xi$ & 0.4966 & 0.0034 & 0.0349 & 0.0350 & 0.0279 \\
\hline & $\sigma$ & 1.5062 & -0.0062 & 0.3376 & 0.3376 & 0.2689 \\
\hline
\end{tabular}

\section{2 Application to Real Survival and Reliability Data}

Here, we present two applications to real data to illustrate the potentiality of the $\mathcal{G} \mathcal{G P}$ model. To compare the performance of the $G \mathcal{G P}$ model, we use two generalizations of the $G \mathcal{P}$ distribution well-established in the literature. We consider the four-parameter beta generalized Pareto $(\mathcal{B G P})$ model proposed by (Mahmoudi, E., 2011) and the Kumaraswamy generalized Pareto $(\mathcal{K} G \mathcal{P})$ model pioneered by (Nadarajah, S. \& Eljabri, S., 2013). The density functions of the $\mathcal{K} \mathcal{G P}$ and $\mathcal{B G P}$ models are given by

$$
\pi_{\mathcal{K G} \mathcal{P}}(x ; \xi, \sigma, a, b)=\frac{a b}{\sigma} z^{-1 / \xi-1}\left(1-z^{-1 / \xi}\right)^{a-1}\left[1-\left(1-z^{-1 / \xi}\right)^{a}\right]^{b-1}
$$

and

$$
\pi_{\mathcal{B G P}}(x ; \xi, \sigma, \alpha, \beta)=\frac{z^{-\beta / \xi-1}\left(1-z^{-1 / \xi}\right)^{\alpha-1}}{\sigma \mathcal{B}(\alpha, \beta)},
$$

respectively, where $z=1+\xi x / \sigma$ and $\mathcal{B}(\cdot, \cdot)$ is the beta function.

Setting $z^{-1 / \xi}=u$ in equation (20) corresponds to equation (15) of (Nadarajah, S.\& Eljabri, S., 2013) with $t=0$. Equation (21) corresponds to the equivalent parameterization considered in equation (5) of (Mahmoudi, E., 2011) with $\mu=0$ and $-\xi$ in place of $\xi$.

The first data set was obtained in (Proschan, F., 1963) and corresponds to the time of successive failures of the air conditioning system of jet airplanes. These data were also studied by (Dahiya, R. C. \& Gurland, J., 1972; Gleser, L. J., 1989; Gupta, R. D. \& Kundu, D.,2001; Kuş, C., 2007), among others. The data are: 194, 413, 90, 74, 55, 23, 97, 50, 359, $50,130,487,102,15,14,10,57,320,261,51,44,9,254,493,18,209,41,58,60,48,56,87,11,102,12,5,100,14$, $29,37,186,29,104,7,4,72,270,283,7,57,33,100,61,502,220,120,141,22,603,35,98,54,181,65,49,12,239$, $14,18,39,3,12,5,32,9,14,70,47,62,142,3,104,85,67,169,24,21,246,47,68,15,2,91,59,447,56,29,176,225$, $77,197,438,43,134,184,20,386,182,71,80,188,230,152,36,79,59,33,246,1,79,3,27,201,84,27,21,16,88$, 
$130,14,118,44,15,42,106,46,230,59,153,104,20,206,5,66,34,29,26,35,5,82,5,61,31,118,326,12,54,36$, $34,18,25,120,31,22,18,156,11,216,139,67,310,3,46,210,57,76,14,111,97,62,26,71,39,30,7,44,11,63$, 23, 22, 23, 14, 18, 13, 34, 62, 11, 191, 14, 16, 18, 130, 90, 163, 208, 1, 24, 70, 16, 101, 52, $208,95$.

Table presents some descriptive statistics for these data. In Table, we provide the MLEs (and the corresponding standard errors in parentheses) of the fitted models and also the values of the following statistics: Akaike information criterion (AIC), Bayesian information criterion (BIC) and consistent Akaike information criterion (CAIC). Based on these results, we note that the $\mathcal{G G P}$ model has the lowest values of the AIC, BIC and CAIC statistics, so this model could be chosen as the best model.

Table 2. Descriptives statistics for the air conditioning system of airplanes data

\begin{tabular}{lr}
\hline Statistic & \\
\hline Mean & 93.141 \\
Median & 57 \\
Variance & 11398.471 \\
Minimum & 1 \\
Maximum & 603 \\
\hline
\end{tabular}

Table 3. MLEs (standard errors in parentheses) and the AIC, BIC and CAIC statistics for the air conditioning system of airplanes data

\begin{tabular}{lccccccc} 
Distribution & $\widehat{\xi}$ & $\widehat{\sigma}$ & $\widehat{a}$ & $\widehat{b}$ & AIC & BIC & CAIC \\
\hline $\mathcal{G G P}$ & 0.482 & 24.386 & 1.745 & - & $\mathbf{2 3 6 4 . 2 0 9}$ & $\mathbf{2 3 7 4 . 2 9 2}$ & $\mathbf{2 3 6 4 . 3 2 3}$ \\
& $(0.070)$ & $(2.176)$ & $(0.117)$ & - & & & \\
$\mathcal{B} \mathcal{G P}$ & 9.889 & 1.452 & 19.200 & 24.535 & 2388.909 & 2402.354 & 2389.101 \\
& $(0.574)$ & $(0.347)$ & $(0.609)$ & $(0.765)$ & & & \\
$\mathcal{K} \mathcal{G P}$ & 5.419 & 1.019 & 8.887 & 36.257 & 2366.357 & 2379.802 & 2366.549 \\
& $(0.317)$ & $(0.372)$ & $(0.953)$ & $(1.861)$ & & & \\
\hline
\end{tabular}

Plots of the pdf and cdf of the fitted $\mathcal{G G P}, \mathcal{K} \mathcal{P P}$ and $\mathcal{B} \mathcal{P}$ models to the air conditioning system of airplanes data are displayed in Figure. These plots seem to indicate that the fitted $\mathcal{G} \mathcal{P P}$ model is the best one in terms of fit to the current data.

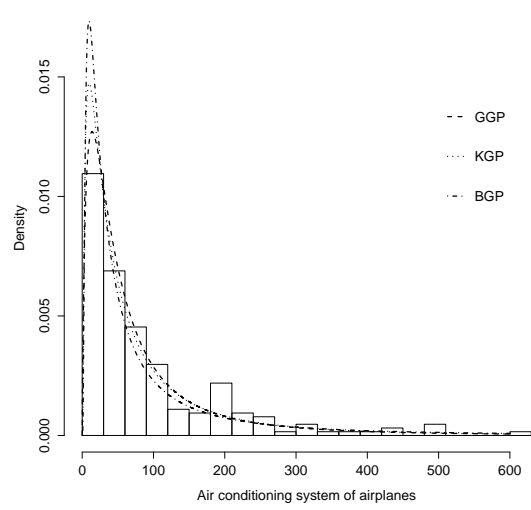

(i)

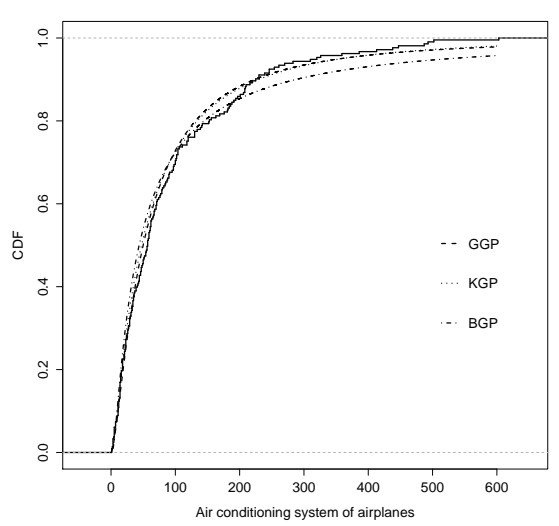

(j)

Figure 3. (a) Plots of the fitted $\mathcal{G G P}, \mathcal{K} \mathcal{G P}$ and $\mathcal{B G P}$ densities; (b) Plots of the estimated cdfs of the $\mathcal{G} \mathcal{G P}, \mathcal{K} \mathcal{P P}$ and $\mathcal{B G P}$ models. .

The formal tests of the goodness-of-fit to the air conditioning system of airplanes data are given in Table . We consider the values of the Kolmogorov-Smirnov $(\mathrm{K}-\mathrm{S})$, Cramér-von Mises $\left(\mathrm{W}^{*}\right)$ and Anderson-Darling $\left(\mathrm{A}^{*}\right)$ statistics. In general, the best fit to the data refers to the model with the lowest value of these statistics. Based on these results, we conclude that the fitted $\mathcal{G} \mathcal{P P}$ model is superior to the other fitted models to these data. 
Table 4. Goodness-of-fit tests for the $\mathcal{G G P}, \mathcal{B} \mathcal{P P}$ and $\mathcal{K} \mathcal{G P}$ models for the air conditioning system of airplanes data

\begin{tabular}{lccc}
\hline \multirow{2}{*}{ Model } & \multicolumn{3}{c}{ Statistics } \\
\cline { 2 - 4 } & $\mathrm{K}-\mathrm{S}$ & $\mathrm{W}^{*}$ & $\mathrm{~A}^{*}$ \\
\hline $\mathcal{G G P}$ & $\mathbf{0 . 0 5 3}$ & $\mathbf{0 . 0 9 3}$ & $\mathbf{0 . 6 6 0}$ \\
$\mathcal{B G P}$ & 0.089 & 0.321 & 2.166 \\
$\mathcal{K} \mathcal{G P}$ & 0.067 & 0.095 & 0.699 \\
\hline
\end{tabular}

The second data set was obtained from Murthy, D.(2004), and consists of the times between failures for repairable items: $1.43,0.11,0.71,0.77,2.63,1.49,3.46,2.46,0.59,0.74,1.23,0.94,4.36,0.40,1.74,4.73,2.23,0.45,0.70,1.06,1.46$, $0.30,1.82,2.37,0.63,1.23,1.24,1.97,1.86,1.17$. Table gives some descriptive statistics for these data. In Table, we present the MLEs (and the corresponding standard errors in parentheses) of the fitted models and also the values of the AIC, BIC and CAIC statistics. Based on these results, it is noted that the GGP model has the lowest values of the AIC, BIC and CAIC statistics, so it could be chosen as the best model to the these data.

Table 5. Descriptives statistics for the times between failures

\begin{tabular}{ll}
\hline Statistic & \\
\hline Mean & 1.543 \\
Median & 1.235 \\
Variance & 1.272 \\
Minimum & 0.110 \\
Maximum & 4.730 \\
\hline
\end{tabular}

Table 6. MLEs (standard errors in parentheses) and the AIC, BIC and CAIC statistics for the times between failures

\begin{tabular}{lccccccc} 
Distribution & $\widehat{\xi}$ & $\widehat{\sigma}$ & $\widehat{a}$ & $\widehat{b}$ & AIC & BIC & CAIC \\
\hline $\mathcal{G G P}$ & 0.028 & 0.698 & 2.100 & - & $\mathbf{8 5 . 2 5 2}$ & $\mathbf{8 9 . 4 5 5}$ & $\mathbf{8 6 . 1 7 5}$ \\
& $(0.170)$ & $(0.449)$ & $(0.817)$ & - & & & \\
$\mathcal{B} \mathcal{G P}$ & 0.156 & 11.061 & 1.979 & 13.850 & 87.257 & 92.862 & 88.857 \\
& $(1.960)$ & $(3.852)$ & $(0.460)$ & $(1.983)$ & & & \\
$\mathcal{K} \mathcal{G P}$ & 1.119 & 4.972 & 1.917 & 14.432 & 87.283 & 92.888 & 88.883 \\
& $(2.371)$ & $(4.817)$ & $(0.837)$ & $(12.687)$ & & & \\
\hline
\end{tabular}

Plots of the fitted $\mathcal{G G P}, \mathcal{K} \mathcal{G P}$ and $\mathcal{B G P}$ models to the times between failures are displayed in Figure for the densities and cumulative functions. Based on these plots, these models seem equivalent in terms of fit to the data. In Table, we present formal goodness-of-fit tests to the times between failures. The values of the K-S, $\mathrm{W}^{*}$ and $\mathrm{A}^{*}$ statistics indicate that the fitted $\mathcal{G G P}$ and $\mathcal{K} G \mathcal{P}$ models are competitive models to these data, although the $\mathcal{G} \mathcal{G P}$ model has less parameters.

Table 7. Goodness-of-fit tests for the $\mathcal{G} \mathcal{G P}, \mathcal{B G P}$ and $\mathcal{K} \mathcal{G P}$ models for the times between failures

\begin{tabular}{lccc}
\hline \multirow{2}{*}{ Model } & \multicolumn{3}{c}{ Statistics } \\
\cline { 2 - 4 } & $\mathrm{K}-\mathrm{S}$ & $\mathrm{W}^{*}$ & $\mathrm{~A}^{*}$ \\
\hline $\mathcal{G} \mathcal{G P}$ & $\mathbf{0 . 0 6 5}$ & $\mathbf{0 . 0 1 8}$ & $\mathbf{0 . 1 3 4}$ \\
$\mathcal{B} G \mathcal{P}$ & 0.066 & 0.018 & 0.140 \\
$\mathcal{K} \mathcal{G P}$ & 0.070 & 0.017 & 0.128 \\
\hline
\end{tabular}

In summary, we conclude that the fitted $\mathcal{G} \mathcal{P P}$ model shows superior performance to other competitive fitted models for two real data sets with the advantage of having less parameters.

\section{Conclusion}

In this paper, we study a three-parameter model named gamma generalized Pareto (GGP) distribution, which consists of a major extension of the generalized Pareto (GP) distribution pioneered by (Pickands, J., 1975). We give some results 


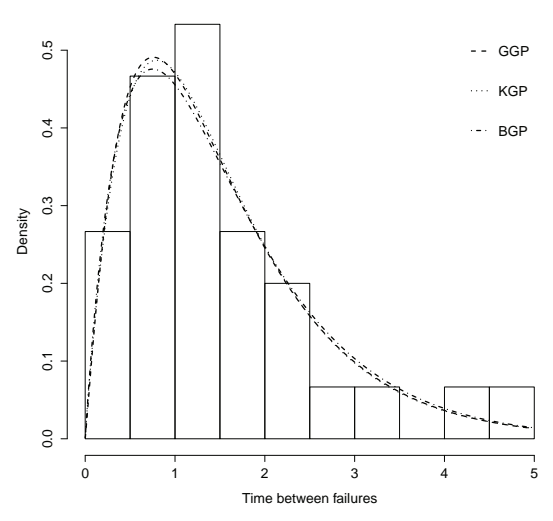

(k)

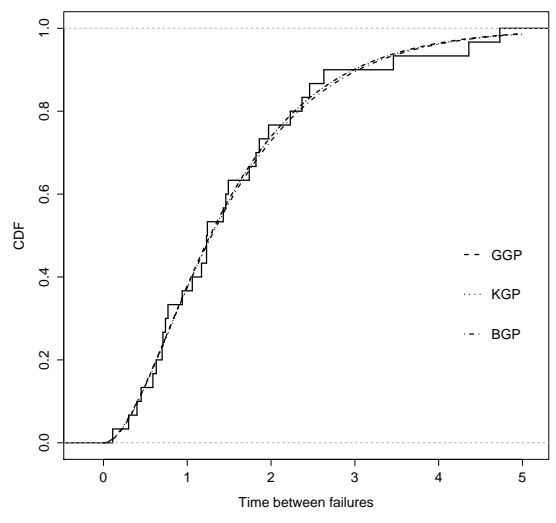

(1)

Figure 4. (a) Plots of the fitted $\mathcal{G G P}, \mathcal{K} \mathcal{G P}$ and $\mathcal{B G P}$ densities; (b) Plots of the estimated cdfs of the $\mathcal{G G P}, \mathcal{K} \mathcal{G P}$ and $\mathcal{B G P}$ models. .

connecting the $\mathcal{G} \mathcal{G P}$ model with other well-established distributions in the literature, such as the gamma and log-gamma distributions. We also provide several mathematical properties of the $G \mathcal{G P}$ model including explicit expressions for the density and quantile functions, ordinary and incomplete moments, mean deviations, Bonferroni and Lorenz curves, generating function, Rényi entropy and order statistics. We discuss the maximum likelihood method to estimate the model parameters and provide the elements of the score vector and the observed information matrix. We also present a Monte Carlo simulation study to evaluate the performance of the maximum likelihood estimators for the $\mathcal{G G P}$ model. Finally, two applications illustrate the potentiality of the $\mathcal{G G P}$ distribution to fit survival data.

\section{References}

Adamidis, K., \& Loukas, S. (1998). A lifetime distribution with decreasing failure rate. Statistics $\mathcal{E}$ Probability Letters, $39,35 \mathrm{C} 42$.

Alzaatreh, A., Famoye, F., \& Lee, C. (2012). GammaCPareto Distribution and Its Applications. Journal of Modern Applied Statistical Methods 11, 78C94.

Carrasco, J. M. F., Ortega, E. M. M., \& Cordeiro, G. M. (2008). A generalized modifiedWeibull distribution for lifetime modeling. Computational Statistics $\mathcal{E}$ Data Analysis, 53, 450C462.

Cordeiro, G. M. (2013). A simple formula based on quantiles for the moments of beta generalized distributions. Journal of Statistical Computation and Simulation, 83, 1932C1943.

Cordeiro, G. M., \& de Castro, M. (2011). A new family of generalized distributions. Journal of Statistical Computation and Simulation, 81, 883C893.

Dahiya, R. C., \& Gurland, J. (1972). Goodness of fit tests for the gamma and exponential distributions. Technometrics, 14, 791C801.

De Zea Bermudez, P., \& Kotz, S. (2010). Parameter estimation of the generalized Pareto distribution-Part I. Journal of Statistical Planning and Inference, 140, 1353C1373.

Eugene, N., Lee, C., \& Famoye, F. (2002). Beta-normal distribution and its applications. Communications in Statistics Theory and Methods, 31, 497C512.

Gleser, L. J. (1989). The gamma distribution as a mixture of exponential distributions. The American Statistician, 43, $115 \mathrm{C} 117$.

Gupta, R., Gupta, P., \& Gupta, R.(1998). Modeling failure time data by Lehman alternatives. Communications in Statistics - Theory and Methods, 27, 887C904.

Gupta, R. D., \& Kundu, D. (2001). Exponentiated Exponential Family: An Alternative to Gamma and Weibull Distributions. Biometrical Journal, 43, 117C130.

Kenney, J. F., \& Keeping, E. S. (1962). Mathematics of Statistics. 3rd ed., Part 1, New Jersey.

Kuş, C. (2007). A New Lifetime Distribution. Computational Statistics $\mathcal{E}$ Data Analysis, 51, 4497C4509. 
Lehmann, E. L. (1953). The power of Rank tests. Annals of Mathematical Statistics, 24, 23C43. https://doi.org/10.1080/00401706.1963.10490105

Lemonte, A. J., \& Cordeiro, G. M. (2011). The exponentiated generalized inverse gaussian distribution. Statistics $\mathcal{E}$ Probability Letters, 81, 506C517.

Mahmoudi, E. (2011). The beta generalized Pareto distribution with application to lifetime data. Mathematics and Computers in Simulation, 81, 2414C2430.

Marshall, A. W., \& Olkin, I. (1997). A new method for adding a parameter to a family of distributions with applications to the exponential and Weibull families. Biometrika, 84, 641C652.

Moors, J. J. (1988). A quantile alternative for kurtosis. Journal of the Royal Statistical Society: Series D, 37, 25 C32.

Mudholkar, G. S., \& Srivastava, D. K. (1993). Exponentiated Weibull family for analyzing bathtub failure rate data. IEEE Transactions on Reliability, 42, 299C302.

Murthy, D., Xie, M., \& Jiang, R. (2004). Weibull models. Wiley series in probability and statistics. Hoboken: John Wiley $\&$ Sons, New York,

Nadarajah, S., Cordeiro, G. M., \& Ortega, E. M. M. (2013). The Gamma-G Family of Distributions: Mathematical Properties and Applications. Communications in Statistics - Theory and Methods. To Appear.

Nadarajah, S., \& Eljabri, S.(2013). The Kumaraswamy GP Distribution. Journal of Data Science, 11, 739C766.

Nadarajah, S., \& Gupta, A. K. (2007). The exponentiated gamma distribution with application to drought data. Calcutta Statistical Association Bulletin, 59, 29C54.

Nadarajah, S., \& Kotz, S. (2003). The exponentiated Frechet distribution. Interstat. Available online from interstat. statjournals.net/YEAR/2003/articles/0312001.pdf

Nascimento, D. C., Bourguignon, M., ..., \& Cordeiro, G. M. (2014). The gamma extended Weibull family of distributions. Journal of Statistical Theory and Applications, 13, $1 \mathrm{C} 16$.

Pickands, J. (1975). Statistical inference using extreme order statistics. Annals of Statistics, 3, 119C131.

Pinho, L. G., Cordeiro, G. M., \& Nobre, J. S. (2012). The GammaCExponentiated Weibull Distribution. Journal of Statistical Theory and Applications, 11, 379C395.

Proschan, F. (1963). Theoretical Explanation of Observed Decreasing Failure Rate. Technometrics, 5, 375C383. https://doi.org/10.1080/00401706.1963.10490105

Ristić, M. M., \& Balakrishnan, N. (2012). The gamma-exponentiated exponential distribution. Journal of Statistical Computation and Simulation, 82, 1191C1206.

Song, J., \& Song, S. (2012). A quantile estimation for massive data with generalized Pareto distribution. Computational Statistics $\mathcal{E}$ Data Analysis, 56, 143C150.

Tahir, M., \& Nadarajah, S. (2013). Parameter induction in continuous univariate distributions - Part I: Well-established G-classes. Communications in Statistics - Theory and Methods. To Appear.

Zografos, K., \& Balakrishnan, N. (2009). On families of beta- and generalized gamma-generated distributions and associated inference. Statistical Methodology, 6, 344C362.

\section{Appendix}

We give below the elements of the observed information matrix of the $\mathcal{G} \mathcal{G P}$ model:

$$
\begin{aligned}
\frac{\partial^{2} \ell(\boldsymbol{\theta})}{\partial \xi^{2}}= & \frac{n(a-1)}{\xi^{2}}-\frac{2}{\xi^{2}} \sum_{i=1}^{n} \log \left(z_{i}\right)-\frac{(1+\xi)}{\xi^{3}} \sum_{i=1}^{n} \frac{\left(z_{i}-1\right)^{2}}{z_{i}^{2}}+\frac{2}{\xi^{3}} \sum_{i=1}^{n} \frac{\left(z_{i}-1\right)}{z_{i}} \\
& -\frac{(a-1)}{\xi^{2}} \sum_{i=1}^{n} \frac{\left(z_{i}-1\right)^{2}}{z_{i}^{2} \log \left(z_{i}\right)}\left(1+\frac{1}{\log \left(z_{i}\right)}\right), \\
\frac{\partial^{2} \ell(\boldsymbol{\theta})}{\partial \sigma^{2}}= & \frac{n}{2}-\frac{(a-1)}{\sigma^{2}} \sum_{i=1}^{n}\left[\frac{\left(z_{i}-1\right)^{2}}{\left[z_{i} \log \left(z_{i}\right)\right]^{2}}+\frac{\left(z_{i}-1\right)^{2}}{z_{i}^{2} \log \left(z_{i}\right)}-\frac{2\left(z_{i}-1\right)}{z_{i} \log \left(z_{i}\right)}\right]
\end{aligned}
$$




$$
\begin{aligned}
& +\frac{(1+\xi)}{\xi \sigma^{2}} \sum_{i=1}^{n}\left[\frac{\left(z_{i}-1\right)^{2}}{z_{i}^{2}}-\frac{2\left(z_{i}-1\right)}{z_{i}}\right] \\
\frac{\partial^{2} \ell(\boldsymbol{\theta})}{\partial \xi \partial \sigma}= & -\frac{1}{\xi^{2} \sigma} \sum_{i=1}^{n} \frac{\left(z_{i}-1\right)}{z_{i}}-\frac{(1+\xi)}{\xi^{2} \sigma} \sum_{i=1}^{n}\left[\frac{\left(z_{i}-1\right)^{2}}{z_{i}^{2}}-\frac{\left(z_{i}-1\right)}{z_{i}}\right] \\
& +\frac{(a-1)}{\xi \sigma} \sum_{i=1}^{n}\left[\frac{\left(z_{i}-1\right)^{2}}{\left[z_{i} \log \left(z_{i}\right)\right]^{2}}+\frac{\left(z_{i}-1\right)^{2}}{z_{i}^{2} \log \left(z_{i}\right)}-\frac{z_{i}-1}{z_{i} \log \left(z_{i}\right)}\right] \\
\frac{\partial^{2} \ell(\boldsymbol{\theta})}{\partial \xi \partial a}= & -\frac{n}{\xi}+\frac{1}{\xi} \sum_{i=1}^{n} \frac{\left(z_{i}-1\right)}{z_{i} \log \left(z_{i}\right)} \\
\frac{\partial^{2} \ell(\boldsymbol{\theta})}{\partial a \partial \sigma}= & -\frac{1}{\sigma} \sum_{i=1}^{n} \frac{\left(z_{i}-1\right)}{z_{i} \log \left(z_{i}\right)} \\
\frac{\partial^{2} \ell(\boldsymbol{\theta})}{\partial a^{2}}= & -n \psi^{\prime}(a)
\end{aligned}
$$

where $\psi^{\prime}(a)=\partial^{2} \log [\Gamma(a)] / \partial a^{2}$ and $z_{i}=1+\xi x_{i} / \sigma$.

\section{Copyrights}

Copyright for this article is retained by the author(s), with first publication rights granted to the journal.

This is an open-access article distributed under the terms and conditions of the Creative Commons Attribution license (http://creativecommons.org/licenses/by/4.0/). 